\title{
Bibliografía castellana sobre Teilhard de Chardin (1950-1970)
}

A partir de la eclosión del "fenómeno" Teilhard en 1955, pero sobre todo, desde 1963, la figura y la obra del genial jesuita francés ha ocupado un puesto central en la atención de investigadores y divulgadores, hasta tal punto que ha podido hablarse ya de "la década Teilhard de Chardin".

Ciertamente, ha sido un fenómeno universal, debido a muy complejas causas, que no es momento de analizar aquí. Baste el dato significativo de que sus principales obras, a sólo diez años de su publicación original, estaban traducidas a la totalidad de las lenguas cultas, incluidos el ruso, japonés, el chino y el árabe.

Por supuesto, esta eclosión teilhardiana no alcanzó la misma intensidad en todas partes. Ha sido el gran tema central de referencia en el área cultural francesa (Francia, Bélgica, Canadá). Pero, contra todo pronóstico aparente, se difundió muy pronto también por las áreas sajona (Inglaterra, Estados Unidos, Holanda) e hispana (España, Portugal y Latinoamérica), al mismo tiempo que penetraba también con fuerza en la Europa del Este (Polonia, Hungría, Rumanía). Finalmente, de modo lento pero progresivo, alcanzó la cerrada área germánica y rompía el hermetismo secular de la cultura arábiga (aunque débilmente).

Concretándome a España y sirviéndome de las publicaciones (libros y revistas, originales o versiones) como criterio orientador, la influencia teilhardiana se ajusta a la curva resultante de la siguiente estadística aproximativa:

$\begin{array}{rrrrrrrr}\text { hasta } & 1958 & \ldots & 16 & & 1964 & \ldots & 26 \\ 1959 & \ldots & 8 & & 1965 & \ldots & 57 \\ 1960 & \ldots & 11 & & 1966 & \ldots & 47 \\ 1961 & \ldots & 6 & & 1967 & \ldots & 44 \\ 1962 & \ldots & 10 & & 1968 & \ldots & 36 \\ 1963 & \ldots & 24 & & 1969 & \ldots & 24\end{array}$

A partir de 1963 en que cobra fuerza, el "fenómeno" Teilhar alcanza rápidamente su cima en el área castellana (1965), para iniciar luego 
un suave, pero progresivo descenso, que se acentúa ya marcadamente en 1969 (aunque los datos para este año son incompletos).

Pero es preciso tener en cuenta un dato de gran interés: el elevado número de traducciones, sobre todo por lo que respecta a los libros, de los que constituyen un $75 \%$. Esto nos revela el carácter "colonizador" de la influencia teilhardiana en España e Hispanoamérica. Lo que se ajusta, por lo demás, al índice general de nuestra actual situación religiosocultural. No obstante, tras la labor divulgadora de Crusafont y Colomer, contamos con las obras valiosas de Riaza, Bravo y Sahagún Lucas, que pueden significar un anticipo de otros estudios en gestación. Porque, en definitiva, tras la oleada del teilhardismo $-\mathrm{y}$ del antiteilhardismo-, queda incólume la presencia de Teilhard de Chardin que sólo ahora, apaciguada un tanto la polémica, podrá ser estudiado en serenidad y profundidad.

Mientras, Teilhard de Chardin se nos está convirtiendo en un "clásico". Por eso, tras la llamada de su descubrimiento masivo, queda todavía la influencia infinitamente más profunda y duradera de un pensamiento tan revolucionario como catalizador, de cara a la nueva cosmovisión y al nuevo humanismo que se gesta en este mismo momento. .

(N. B. El. asterisco indica que se trata de una traducción).

1. Obras traducidas al castellano.

El Fenómeno humano. Taurus. Madrid 1963 (4. $\left.{ }^{\mathrm{a}}, 1967\right)$

La Aparición del hombre. Ib., 1958 (6. ${ }^{\mathrm{a}}$ 1967).

La Visión del pasado. Ib. 1958 (6. ${ }^{\mathrm{a}} 1967$.

El Medio divino. Ib., 1959 (6. 1967).

El Porvenir del hombre. Ib., 1962 (4. ${ }^{a}$ 1967).

La Energía humana. Ib., 1963 (2. ${ }^{\text {a } 1967) .}$

La activación de la energía. Ib., 1965 (2. 1967$).$

El grupo zoológico humano. Ib., 1957 (5. ${ }^{\mathrm{a}}$ 1967).

Ciencia y Cristo. Ib., 1968.

Cartas de viaje (1923-1939). Ib., 1957 (4. ${ }^{a}$ 1966).

Nuevas cartas de viaje (1939-1955). Ib., 1960 (4. ${ }^{\mathrm{a}}$ 1967).

Escritos del tiempo de la guerra. Ib., 1966 (2.a 1967).

Génesis de un pensamiento. Ib., 1963 (2. ${ }^{2}$ 1965).

Cartas de Egipto. Ib., 1967.

Terminado ya este trabajo ha llegado a nuestro poder la obra de Joan E. JARQUE, Bibllographie générale des Ouvres et Articles sur Pierre Teilhard de Chardin parus jusqu'à fin décembre 1969. Ed. Universitaires, Fribourg/Suiza 1970, 206 pp.

Se trata de una compilación bibliográfica que intenta ser exhaustiva y que resulta serlo casi siempre. Por lo tanto, incluye ya la mayoría de los libros y artículos que aqui presentamos, pero presenta algunas lagunas graves, sobre todo por lo que respecta a libros originales (omite los libros de F. Riaza y E. Guerrero) y, especialmente. a los traducidos. También comete la anomalla de citạr a un mismo autor, Juan Sahagún Lucas Hernández, por las tres referencias independientes de Sahagún, Lucas y Hernández. Para más detalles véase recensión en este mismo artículo. 
Cartas de Hastings y de París. Ib., 1968

Himno del Universo. Ib., 1964.

Yo me explico. Ib., 1968.

Cómo creo yo. Ib. 1970.

PTC. Imágenes y palabras. Ib. Madrid, 1967, $224 \mathrm{pp}$.

Blondel y Teilhard de Chardin, Hechos y Dichos, Zaragoza 1968.

\section{Libros.}

BALLESTER ESCALAS, R., Teilhard de Chardin. Toray, Barcelona 1967, $214 \mathrm{pp}$.

BAYCE, B., Aproximación a TC. Arca, Montevideo 1965, 92 pp.

* BORDET, CH., La actualidad del mensaje de TC. Betis, Barcelona $1968,120 \mathrm{pp}$.

BRAVO, F., TC. Su concepción de la historia. Nova Terra, Barcelona, 1970, $435 \mathrm{pp}$.

BRIONES TOLEDO, A., PTC y otros ensayos. A. Bello, Santiago de Chile 1966, $149 \mathrm{pp}$.

COLOMER, E., Mundo y Dios al encuentro. El evolucionismo cristiano de PTC Nova Terra, Barcelona, 1963. 142 pp. 1964, 148 pp.

* CORVEz, M., De la ciencia a la fe. TC. Mensajero, Bilbao, 1970, 152 pp.

* CRESPY, G., Ensayo sobre TC. De la ciencia a la teología. Sígueme, Salamanca, 1967, $227 \mathrm{pp}$.

CRUSAFONT, M., Evolución y ascensión (Ensayos en la línea TC). Taurus, Madrid, 1960, $122 \mathrm{pp}$.

CRUSAFONT, M., Origen, evolución y singularidad del hombre (Idem). Taurus, Madrid, 1965, $90 \mathrm{pp}$.

CRUSAFONT, M., El fenómeno vital (Idem). Bercelona, 1967, $132 \mathrm{pp}$.

CRUSAFONT, M., El fenómeno humano según TC (Conferencia) Sabadell, 1956, $24 \mathrm{pp}$.

CRUSAFONT, M., El nuevo humanismo trascendente del PTC (Conferencia). Barcelona 1960, $18 \mathrm{pp}$.

* CUENOT, C., PTC Las grandes etapas de su evolución, Taurus, Madrid 1967, $627 \mathrm{pp}$ (La versión castellana supera a la original).

* CUENOT, C., Teilhard de Chardin. Labor, Barcelona. 1966, 216 pp.

* CUENOT, C., Nuevo Léxico de TC. Taurus, Madrid 1970, 310 pp.

* CUYPERS, H., Por y contra Teilhard. Columba, Buenos Aires 1968, $84 . \mathrm{pp}$.

* CUYPERS, H., Vocabulario TC. Columba, Buenos Aires 1968, 136 pp.

CUYPERS, H., Presencia de TC. (En colaboración con MAGLOIRE). Betis, Barcelona, 1967, $260 \mathrm{pp}$.

* CHAUCHARD, P., El ser humano según TC, Herder, Barcelona, 1966, $226 \mathrm{pp}$.

* Chauchard, P., el pensamiento científico de TC. Península, Barcelona, 1966, $262 \mathrm{pp}$. 
* CHAUChaRD, P., Teilhard y el optimismo de la cruz. Columba, Buenos Aires 1968, $84 \mathrm{pp}$.

* CHAUChaRd, P., Teilhard testigo de amor Columba. Buenos Aires, 1968, $64 \mathrm{pp}$.

* DELFGAAUW, B., TC y el problema de la evolución. Lohlé, Buenos Aires, 1966, $126 \mathrm{pp}$.

* DEVAUX, A., T y la vocación de la mujer. Columba, Buenos Aires, 1968. $84 \mathrm{pp}$.

* DEVAUX, A., T y Saint-Exupéry. Convergencias $y$ divergencias. Columba, Buenos Aires, 1968. $108 \mathrm{pp}$.

* DOMENACH, J.- M., TC y el personalismo. Nova Terra, Barcelona, 1969, $162 \mathrm{pp}$.

* DUROUX, P.- E., Historia natural de la humanidad según T. Columba, Buenos Aires, 1968, $134 \mathrm{pp}$.

* GEORGE, N. De Einstein a Teilhard. Betis, Barcelona, 1966, 230 pp.

* GOSZTONYI, El hombre y la evolución. La antropología filosófica de TC. Studium, Madrid, 1970,232 pp.

* GRENET, P. B., TC, filósofo a pesar suyo, Ed. Paulinas, Buenos Aires 1965. $252 \mathrm{pp}$.

* GRENET, P. B., Teilhard de Chardin. Halar, Madrid 1962, 218 pp.

* GRENET, P. B., TC, un evolucionista cristiano. El Cid, Madrid, 1965, $220 \mathrm{pp}$.

GUERRA, L. F., La filosofía de la naturaleza en TC. Rlva-Agüero, Lima, $120 \mathrm{pp}$.

GUERRERO, E., TC. Aspectos fundamentales de su obra. Studium, Madrid, 1969, $174 \mathrm{pp}$.

* HEER, F., y otros, La nueva imagen del hombre en TC. Guadalupe, Buenos Aires. 1967, 71 pp.

* KOPP, J. V.. Oricen y futuro del hombre. TC y su concepción del mundo. Herder, Bercelona. 1965, 105 pp.

* LAFAY, G., T C. Sintesis de su pensamiento. Sígueme, Salamanca 1967, 76 pp. (más esquemas)

* LEON - DUFOUR, L., TC y el problema del porvenir del hombre. Taurus, Madrid, 1969, 107 pp.

* LEPP, I., La nueva tierra. TC y el cristianismo en el mundo moderno. Iohlé, Buenos Aires, 1963, 200 pp.

* LEROY, P., Perfil humano de TC, Nova Terra, Barcelona, 1965, 56 pp.

* LIGNEUL, A., Teilhard $y$ el personalismo. Columba, Buenos Aires, 1968, $96 \mathrm{pp}$.

* LUBAC, H. de., La oración de Teilhard. Estela, Barcelona, 1966, 228 pp.

* LUBAC, $\mathrm{H}$. de., El pensamiento religioso de TC. Taurus, 1967, $456 \mathrm{pp}$.

* LUBAC, H. de., Blondel y TC. Hechos y dichos. Zaragoza 1968, 208 pp.

* LUBAC, H. de., TC en diálogo con el hombre de hoy. Hechos y Dichos. Zaragoza 1968, $144 \mathrm{pp}$.

* LUBAC, H. de., El eterno femenino. Sígueme, Salamanca 1969, 287 pp. 
LUCAS, J. S., La unificación de los seres en TC (extracto de tesis doctoral) Verdad y Vida, Madrid, 1968, 48 pp.

LUCAS, J. S., El hombre social en el pensamiento de TC. Fontanella, Barcelona, 1969, $207 \mathrm{pp}$.

* MAGloire, G. y CUYPers, H. Presencia de TC. Betis, Barcelona, $1967,260 \mathrm{pp}$.

* Maloney, G. A., El Cristo Cósmico de San Pablo a TC. Sal Terrae, Santander 1969, 265 pp. (169-204).

* MARTIN, M. G., La espiritualidad de TC. Perpetuo Socorro, Madrid, 1969.

* MATHIEU, P.L., El pensamiento político y económico de TC. Taurus, Madrid 1970, $291 \mathrm{pp}$.

MEINVIELLE, J., La cosmovisión de TC. Buenos Aires, 1960, 48 pp.

MEINVIELLE, J., PTC o la religión de la evolución Ed. Theoría, Buenos Aires 1965, $282 \mathrm{pp}$.

* MEYER, F., T y las grandes derivas del mundo viviente, Columba, Buenos Aires 1968, $72 \mathrm{pp}$.

* MONESTIER, A. y SAlleRoN, L., En favor y en contra de TC. Pomaire, Santiago de Chile, 1967.

* MOONEY, Ch. F., TC y el misterio de Cristo. Sígueme, Salamanca, 1968, $295 \mathrm{pp}$.

* PERIGORD. M., Evolución y temporalidad en TC. Columba, Buenos Aires 1968, $64 \mathrm{pp}$.

* RABUT, O. A., Diálogo con TC, Estela, Madrid, 1967, 96 pp.

RJA7.A, F., TC y la evolución biolóqica. Alcalá. Madrid, 1968, 433 pp

RIAZA, F., Teilhard $y$ el trabaio, 7.yx. Madrid, 1967, 96 pp.

* RIDEAU E., el pensamiento de TC. Península, Barcelona, 1968, 546 pp.

* RIVIERE, C., En China con TC. Taurus, Madrid 1970.

* SAlleron, L., En favor y en contra de TC (En colaboración con Monestier) Pomaire, Santiago de Chile, 1967.

SANMIGUEL. J., TC. Signo de contradicción. Bilbao, 1967, $162 \mathrm{pp}$.

* SCALTRITI, J., T C. ¿Mito o herejia? Ope, Villalba-Pamplona 1967, $222 \mathrm{pp}$.

* SCHOONENBERG, P., El mundo de Dios en evolución. Lohlé, Buenos Aires, 1966, $154 \mathrm{pp}$. (Estudio libre sobre TC).

* SMUlders, P., La visión de TC. Descleé de Br. Bilbao, 1967, 327 pp.

* THYS, A., Conciencia, reflexión y colectividad en TC. Columba, Buenos Aires 1968, $126 \mathrm{pp}$.

* TRESMONTANT, C. Introducción al pensamiento de TC. Taurus, Madrid 1960, $96 \mathrm{pp}$.

* TRUHLAR, K.V., Soloviev y Teilhard. Poesía y experiencia religiosa. Razón y fe, Madrid 1966, $170 \mathrm{pp.}$

VARIOS (Dubarle, Crusafont, Rof Carballo, F. Riaza, Sanz Criado) En torno a TC. Taurus, Madrid 1969. $128 \mathrm{pp}$.

* VARIOS., Panteismo, Acción, Omega según TC. Marfil, Alcoy 1969, 130 páginas. 
* VARIOS, Evolución, marxismo y cristianismo. Estudio sobre la sintesis de TC. Plaza Janés, Bercelona 1970, $214 \mathrm{pp}$.

* WILDIERS, N.N., Teilhard de Chardin. Fontanella, Barcelona, 1963, $192 \mathrm{pp}$.

\section{(Libros parcialmente dedicados a Teilhard)}

"El teilhardismo", en Historia de la filosofía. La filosofía burguesa contemporánea Grijalbo, México 1966, t. VII.

BRAVO, F., "Cristo en la obra de PTC" en Cristologia y pastoral en América Latina. Nova Terra, Barcelona 1966, 99-206.

COLOMER, E., "Evolución y cristianismo", en La Evolución. BAC. Madrid, 1966, 853-911.

* HILDEBRAND, D. von., "TC: falso profeta" (257-284) y "El teilhardismo", 159-161), en El caballo de Troya en la ciudad de Dios, Fax, Madrjd, 1969.

* MARITAIN, J., El camresino de Garona: "T y el teilhardismo" (163175) y los anejos I y II (335-344).

PARIS, C., "Ser y evolución", En La. Evolución. Ed. Católica, Madrid 1966, 761-791 (sobre Teilhard 782-787).

* PAUPERT, J.- M., ¿Es todavía nosible la fe? El caso $\mathrm{T}$ y la capilla teilhardiana", 184-206. Península, Barcelona 1967.

TOUCEDA, R., Introducción a la socioantronologia (sobre Teilhard 4566) Abeleledo-Perrot, Buenos Aires 1962, $80 \mathrm{pp}$.

VELASCO IBARRA, J.M., Servidumbre $y$ liberación. Del imperialismo atómico a la claridad del esníritu: "Comprender y amar: TC" (129-141). Americalae, Buenos Aires 1965.

\section{Articulos}

"Curs'llos y conferencias" (Madrid 1956). Fascículo III.

"Diez años después" CUADERNOS PARA EL DIALOGO 19 (Madrid 1965) $22-23$.

* "El pensamiento oculto del P. Teilhard" CRISTIANDAD 24 (Barcelona 1967) $188-195$.

"P. Teilhard de Chardin. Su pensamiento en el plano filosófico y religioso" ECCLESIA (Madrid 25-8-1962) 15-18.

"Teilhard, el hombre espiritual" HECHOS Y DICHOS (Zaragoza 1966) 243-246.

"Un 'Monitum' a propósito de la obra de Teilhard de Chardin" ORBIS CATHOLICUS 5 (Barcelona 1962) 415-418.

ABAD, M.A., "La cosmogénesis en Teilhard de Chardin" ENSAYOS 44 "Loyola 1966) 8-12.

AGUIRRE, E., "El legado de P. Teilhard de Chardin" REVISTA DE ANTROPOLOGIA Y ETNOLOGIA, 9 (Madrid 1955) 9-21. 
AGUIRRE, E., Nuevas trayectorias paleontológicas. PROYECION, 7 (Granada 1955) 38-41.

AGUIRRE, E., "El mañana de la evolución" CUADERNOS HISPANOAMERICANOS 65 (Madrid 1966) 5-19.

AGUIRRE, E., Teilhard de Chardin. LA ESTAFETA LITERARIA 280 (Madrid 1963), 2.

ALEMANY, C., "La nueva frontera de la humanidad en Teilhard de Chardin" ENSAYOS 53 (Loyola 1968) 35-41.

ALVAREZ ALVAREZ, J. L., "Teilhard de Chardin ¿profeta o hereje? Una visión objetiva de su pensamiento a través de sus críticos y de sus defensores" VIDA NUEVA 626 (Madrid 1968) 15-22.

ARIAS, I., "Cristo, primer querido y Punto Omega del Universo. El cristocentrismo en Duns Escoto y Teilhard de Chardin" REFLEJOS 26 (León 1965) 59-103.

ARRUPE, P., "Sobre Teilhard de Chardin". CORPORACION 81 (México 1965), 9.

* BaLTHASAR, H.U. von, "La espiritualidad de Teilhard de Chardin" ORBIS CATHOLICUS 7 (Barcelona 1964) 220-237.

BELTRAN DE GUEVARA, J. M., "Teilhard de Chardin o la pasión de síntesis" SIC 28 (Caracas 1965) 174-178.

BENDAÑA R., "Aproximãción al pensamiento ético de Teilhard de Chardin" ESTUDIOS CENTROAMERICANOS 24 (S. Salvador 1969) 229-232.

BENGOECHEA, J.L., "La socialización en Teilhard de Chardin" ENSAYOS 44 (Loyola 1966) 28-30.

BENZO, M., "Evolución y dogma" REV. DE LA UNIV. DE MADRID 29-31 (1959) 533-559.

BLAJOT, J., "La cosmovisión de TC". SEMANA MEDICA 114 (1967), 8-9.

* BRAYBROOKE, N., "C.G. Jung y Teilhard de Chardin. Un diálogo" ARBOR 72 (Madrid 1969) 345-352.

* BRUNNER, A., "La obra de Teilhard de Chardin" ORBIS CATHOLICUS 3 (Barcelona 1960) 300-314.

BULNES, J. M., "El concepto de materia según Teilhard de Chardin" DIALOGOS 2 (Puerto Rico 1965) 87-109.

C. V. M., "Sobre el pensamiento filosófico y teológico de PTC" ESPIRITU 12 Madrid 1963) 31-38.

CACHO, I., "El Cristo cósmico" HECHOS Y DICHOS (Zaragoza 1966) 295-301.

CAMPO ALANGE, M., "Entre biología y humanismo". LA ESTAFETA LITERARIA 290 (Madrid 1964) 5-6.

CANALiS VIDAL, F., "Teilhard de Chardin". CRISTIANDAD 441 (Barcelona 1967) 209.

CASAÑAS, F., "Las visiones de P. Teilhard de Chardin" ILUSTRACION DEL CLERO 54, (Madrid 1961) 297-304.

CASAS, J. A., "Sombras y luces en Teilhard de Chardin" REVISTA JAVERIANA 64 (Bogotá 1965) 142-144. 
CEVALLOS GARCIA, G., "Evocación de Teilhard de Chardin" ANALES DE LA UNIV. DE CUENCA 20 (Cuenca-México 1964) 7-14.

COLOMER, E., "Mundo y Dios al encuentro. El universo cristocéntrico de Teilhard de Chardin" ORBIS CATHOLICUS 5 (Barcelona 1962) 193-215.

COLOMER, E., "La evolución cristocéntrica de Teilhad de Chardin" ESPIRITU 12 (Madrid 1963) 112-126.

COLOMER, E., "En torno a Teilhard de Chardin: la obra y sus intérpretes" SELECCIONES DE LIRBOS Eler, Barcelona 1 (1964) 24-60; 3-4 (1965) 256-264; ib., 276-278; ib., 391-392; 9 (1968) 11-76 (Magnífica orientación bibliográfica).

COLOMER, E., "Teilhard de Chardin, 10 año después" RAZON Y FE 65 (Madrid 1965) 17-36.

COLOMER, E., Diálogo con Teilhard de Chardin" HECHOS Y DICHOS (Zaragoza 1966) 495-497.

COLOMER, E., "El fenómeno Teilhard" HECHOS Y DICHOS (Zaragoza 1966) $282-287$.

COLOMER, E., "Pierre Teilhard de Chardin" ARCHIVUM HISTORICUM SOC. JESU 36 (Roma 1967) 341-367.

COLOMER, E., "Socialización y personalismo en Teilhard de Chardin" CONVIVIUM 28 (Barcelona 1968) 25-52.

COLOMER, E., "En torno a la concepción bíblica y cientifica del hombre. Diálogo entre un teilhardino ilustrado y un teólogo benévolo" HECHOS $¥$ DICHOS (Zaragoża 1969) 215-225.

COLOMER, E., "Teilhard de Chardin y el ateísmo secularista" RAZON Y FE 179 (Madrid 1969) 359-376.

COLOMER, E., y RUBIO, A., "Bibliografía teilhardiana" HECHOS Y DICHó̀ (Zaragoza 1966) 326-336.

COLOMER, E., y otros, "Teilhard de Chardin. Entre origen y destino. Entre la ciencia y la fe. Entre biología y humanismo" LA ESTAFETA LITERARIA (Madrid 25-4-1864) n. $.^{\circ} 22,3-14 ; 23$.

CONGAR, Y., "Iglesia y mundo". CUADERNOS PARA EL DIALOGO 19 (Madrid 1965) 7-11.

* CREN, P., "El cristiano y el munđo según Teilhard de Chardin" CONCILIUM 2/3 (Madrid 1966) 254-266.

CRUSAFONT PAIRO, M., "Antropogénesis y cefalización en el sistema teilhardiano" REV. DE PSIQUIATRIA Y PSICOLOGIA MEDICA 7 (Barcelona 1965) 173-183.

CRUSAFONT; M., "El IV aniversario de la muerte del PTC". BOLETIN INFORMATIVO 17-18 (Sabadell 1959) 2-3.

CRUSAFONT, M., "La hoosfera y el fenómeno humano según las ideas del P. Teilhard de Chardin" ESTUDIOS GEOLOGICOS 17 (Barcelona 1953) $147-153$.

CRUSAFONT, M., "El pensamiento cientifico del P. Teilhard de Chardin" ESTUDIOS GEOLOGICOS 12 (Barcelona 1956) 343-375.

CRUSAFONT, M., "La ley recurrente de complejidad-conciencia al día" 
MEMORIAS de la Real Academia de Ciencias y Artes de Barcelona, 3. época, XXXV, n. 4 (1964) 135-201.

CRUSAFONT, M.,"Antropogénesis y cefalización en el sistema teilhardiano" REV. DE PSICOLOGIA Y PSIQUIATRIA MEDICA VII, n. 3 (Barcelona 1965) 173-183.

CRUSAFONT, M., "Teilhard: compendio, sintesis y convergencia" "HECHOS Y DICHOS (Zaragoza 1966) 247-256.

CRUSAFONT, M., "Concepciones cosmovitalistas del Evolucionismo" MUSEO DE LA CIUDAD DE SABADELL, 4 (Sabadell 1948) 5-28.

CUBILLO, L., "El evólucionismo en Teilhard de Chardin" RELIGION Y CULTURA 10 (Madrid 1965) 353-377.

* CUENOT, C., "Situación de Teilhard de Chardin" ORBIS CATHOLICUS 7 (Barcelona 1964) 193-219.

* CUENOT, C., "Teilhard y el marxismo" CUADERNOS PARA EL DIALOGO 9 (Madrid 1964) 26-29.

* CUENOT, C., "La espiritualidad de Teilhard de Chardin" CUADERNOS PARA EL DIALOGO 17 (Madrid 1965) 10-14.

DALMAU, J., "Teilhard de Chardin y su limitación" EL CIERVO XIV (Barcelona 1965) 12.

* D'ARMAGNAC, C., "El rensamiento de Teilhard de Chardin como apolooetica mcderna" SELECCION!SS DE TEOLOGIA 5 (Madrid 1963) 45-55.

* D'OUINCE, R., "La obediencia en la vida de Teilhard de Chardin" ESTUDTOS CENTROAMERICANOS 19 (S. Salvador 1964) 300-307.

* DEVAUX, A. A., "El hombre de mañana, seoún Teilhard de Chardin" INDICE DE ARTES Y LETRAS 171 (Madrid 1963) 16-17.

DIAZ CORVALAN, E., "La persona en Teilhard de Chardin" BOLETIN IBEROAMERICANO DE INFORMACION 76 (Santiago de Chile 1965) oct., 4-8.

DOLAN, M. E., "Tellhard de Chardin, poeta" CRITERIO 41 (Buenos Aires 1969) 69-72.

* DOLCH, H., "El pecado en un mundo en evolución" CONCILIUM $3 / 2$ Madrid 1967) 415-425.

* DOMENACH, J. M., "El nersonalismo de Teilhard de Chardin" SIC 27 (Caracas 1964) 299-300 y 337-348.

DOMINGUEZ, E., "El sentido humanístico de las carreras técnico-científicas" COMUNIDAD 18 (México 1968) 177-189.

DUSSEL, E., "En torno a la obra de Teilhard de Chardin" CIENCIA Y FE 19 (Buenos Aires 1963) 391-400.

DUSSEL, E., "P. Teihard de Chardin, 'quaestio disputata"' ESTUDIOS 562 (Buenos Aires 1965) 121-132.

EGGERS LAHN, C., "Teilhard de Chardin y el historicismo" ESTUDIOS 562 (Buenos Aires 1965) 107-112.

ESTEBAN ROMERO, A., "El P. Teilhard de Chardin icientiflco optimista y teólogo pesimista? CRISIS 9 (Madrid 1962) 163-180. 
EZCURDIA, A. de: "La obra de Teilhard de Chardin" REVISTA DE LA UNIVERSIDAD DE MEXICO 20 (1966) n..$^{\circ}$ 6. 17-20.

* FAESSLER M., "La experiencia espiritual del P. Teilhard de Chardin" CONFLUENCIAS 1 (Buenos Aires 1964-5) n.. 5, 13-17; n. ${ }^{\circ} 6,11-17$.

* FRENAUD, G., "Estudio crítico sobre el pensamiento filosófico y religioso del P. Teilhard de Chardin" VERBO 17 (Valencia 1963) 58-81; COLLIGITE 3 (León 1963) 83-91; Ed. Iction, Buenos Alres 1967.

* FULlaT, O., "Teilhard de Chardin y Santo Tomás frente al problema de la evolución" CRISIS 6 (Madrid 1959) 61-76.

* FURlong, G., "Carlos Darwin y Teilhard de Chardin" ANALES DE LA ACADEMIA ARGENTINA DE GEOGRAFIA 3 (Buenos Aires 1959) 1519.

GAETE, A., "Teilhard de Chardin: una nueva noción de clencia" ILUSTRACION DEL CLERO 54 (Madrid 1961) 359-364.

GAETE, A., "El descubrimiento de la persona en Teilhard de Chardin" MENSAJE 165 (Santiago de Chile 1967) 664-670.

GAETE, A., "Teilhard de Chardin, ¿entusiasmo o reserva?" MENSAJE 12 (Santiago de Chile 1963) 82-89.

GAETE, A., Introducción al "Fenómeno humano". MENSAJE 145 (1965) $687-692$; 147 (1966) 102-106; 162 (1967) 415-421.

GALEANO, A., "El sentido de la acción en Teilhard de Chardin". FRANCISCANUM 9 (Bogotá 1967) 3-37; 99-120.

* Gat,I, M. von, "P. Teilhard de Chardin, el hombre y el pensador" ORBIS CATHOLICUS 3 (Barcelona 1960) 300-314.

GARCTA ABRIL, G., Bibliografia sobre Teilhard de Chardin" ORBIS CATHOLICUS 7 (Barceona 1964) 238-250.

GARCIA ABRIL, G., "Teilhard de Chardin" ABSIDE 41 (1963) 10-11.

GARCIA ALVAREZ, J., "La nueva visión de universo de Teihard de Chardin" ESTUDIOS FILOSOFICOS 3 (Caldas de Besaya 1964) 253-270.

GIL CREMADES, J. J., "La visión de mundo del P. Teihard de Chardın" NIJESTRO TIEMPO 61 (Pamplona 1959) 21-36.

* GILSON, E., "El 'caso' Teilhard de Chardin" CRISTIANDAD 23 (Barcelona 1966) 31-38; FINIS TERRAE 57 (Santlago de Chile 1966) 3-20.

GOMEZ CAFFARENA, J., "Teilhard y el marxismo" HECHOS Y DICHOS (Zaragoza 1966) n. $.^{\circ} 362,288-294$.

GOMIS, J., "Teilhard de Chardin" EL CIERVO 57 (Barcelona 1957) 8.

GONDRA, J. M., "El 'Fenómeno' de P. Teilhard de Chardin". ENSAYOS 38 (Loyola 1964) 10-13.

GRECORIO DE JESUS, "S. Juan de la Cruz y Teilhard de Chardin" EPHEMERIDES CARMELITICAE 18 (Roma 1967) 362-367.

GUERRA, J., "El evolucionismo de Teilhard de Chardin" COMPOSTELLANUM 2 (Santiago de Compostela 1957) 501-520.

HOURTON, J., "Teilhard de Chardin: ¿ciencia o filosofia?" MAPOCHO 3 (Santiago de Chile 1965) n. 2, 25-36.

HOURTON, J., "Las condiciones de posibilidad de la flosofía cristiana. Irradiación de la posición blondeliana" ANALES DE LA FACULTAD 
DE FILOSOFIA $\mathrm{X}$ CIENCIAS DE LA EDUCACION (Santiago de Chile 1965) 11-36 (Blondel y Teilhard, 29-35).

HOURTON, J., "Teilhard de Chardin y la educación" ANALES DE LA FACULTAD DE FILOSOFIA Y CIENCIAS DE LA EDUCACION (Santiago de Chile 1966) 1-34.

HOYOS VASQUEZ, J., "El método del PTC" REVISTA JAVERIANA 64 (Bogotá 1965) 471-483.

HOYOS VASQUEZ, J., "La fenomenología teilhardiana y la filosofía" Ib., 568-580.

IRIARTE, J., "Al dimidiar este gran siglo nuestro: Lombardi, Przywara, TC" RAZON Y FE 154 (Madrid 1956) 77-91.

JIMENEZ DUQUE, B., "Dios, cosmos, historia". ARBOR 184 (Madrid 1961) $397-420$.

* JOURNET, Ch., "El intento teológico de TC" ARCO 7 (Bogotá 1965) $439-442$.

JUAN, R., "El nuevo humanismo del PTC" SIC 254 (Caracas 1963) 170173.

KUHN, W., "TC y el materialismo dialéctico. Ciencia o metafísica?" ESTUDIOS CENTRO-AMERICANOS 19 (San Salvador 1964) 4-8.

LARA GITARD, A., "En torno a TC" ARBOR 53 (Madrid 1962) 86-87.

LIVI, A., "Etienne Gilson y TC" ARCO 10 (Bogotá 1968) 291-296; idem en NUESTRO TIEMPO 31 (Pamplona 1969) 129-136.

LONDOÑO, A., "Quién era TC?" REVISTA JAVERIANA 63 (Bogotá 1965) 83-93; idem en MENSAJE 14, 90-98; y en ESTUDIOS CENTRO-AMERICANOS 20 (S. Salvador 1965) 194-201.

LOPEZ SALGADO, C., "Metafísica y creación en TC" SAPIENTIA 20 (Buenos Aires 1965) 275-289.

* LUBAC, H. de, "EL PTC, misionero y discípulo de S. Pablo" HECHOS Y DICHOS (Zaragoza 1966) 257-273.

LUCAS, J. S., "Precisiones en torno a Teilhard" VERDAD Y VIDA 25 (Madrid 1967) 535-554.

LUCAS, J.S., "La socialización en el pensamiento de TC" VERDAD Y VIDA 26 (Madrid 1968) 91-134.

LUCAS, J. S., "La evolución de la castidad y el PTC" INCUNABLE 223 (Salamanca 1968). 35-36.

LUCAS, J. S., "En torno a los estudios eclesiásticos. Precisiones del PTC" SEMINARIOS 35 (Salamanca 1968) 275-294.

LUCAS, J. S., "TC y la paz" RAZON Y FE 179 (Madrid 1969) 623-636.

LUJAN, N., "La obra de TC" GLOSA 69 (Barcelona 1960) 23-24 y 124.

LUZZI, J., "TC y el malestar de los teólogos" ESTUDIOS 54 (Buenos Aires 1965) $92-98$.

LUZZI, J., "La percepción del tiempo en TC" CRITERIO 41 (Buenos Aires 1968) $334-340$.

LLANO, A., "Preámbulos para entender a PTC. El fenómeno humano en TC" UNIVERSITAS 29 (Bogotá 1965) 34-57. 
* MACHE, R., "Ciencia, cultura y cristologia. Reflexión de un científico sobre Teilhard" CONCILIUM 4/3 (Madrid 1968) 1708-1717.

* MAGLOIRE, G., "La vida de PTC" POLITICA Y ESPIRITU 280 (Santiago de Chile 1963) 24-29.

MALINOW, C. A., "Finalidad y determinismo en los sistemas evolutivos de PTC y Henri Bergson" DIALOGOS 2 (Puerto Rico 1965) 111-135.

MARQUINEZ ARGOTE, G., "La metafísica que Teilhard rechaza" REISTA JAVERIANA 66 (Bogotá 1966) 386-394.

MARQUINEZ ARGOTE, G., "A qué nivel discurre el pensamiento de TC?" REVISTA JAVERIANA 68 (Bogotá 1967) 373-380.

MARTINEZ SAEZ, S., "Teilhard de Chardin" ISTMO n. 41 (México 1965) 27-32.

MARTINEZ TORRERO, G., "TC, evolución y espiritualidad" SIC 27 (Caracais 1964) $306-309$.

MEINVIELLE, J., "La cosmovisión de TC. Una metafísica del unir" ESTUDIOS FILOSOFICOS Y TEOLOGICOS 2 (Buenos Aires 1960) 107-133.

MEINVIELLE, J., "El marxismo en TC" EL CRUZADO ESPAÑOL 65 (Barcelona 1960) 3-4.

MERIDES CARMELITICAE 18 (Roma 1967) 362-367.

MIGOYA, F.; "El neohumanismo evolutivo de TC" ABSIDE 27 (México 1963) 339-345.

MUÑOZ ALONSO, A., "Entre la ciencia y la fe" LA ESTAFETA LITERARIA (Madrid 25-4-1964) n. ${ }^{\circ}$ 290, 6-7.

MUÑOZ ALONSSO, A., "El teilhardismo en la critica marxista" DIARIO DE CUENCA 26-3-1967.

MUÑ̃̃ ALONSO, A., "Los preliminares teilhardianos de la vida" ANUARIO HUMANITAS 1967 (Universidad de Nuevo León) 173-195.

NEIRA, E., "La respuesta de TC al mundo de hoy" REVISTA JAVERIANA 63 (Bogotá 1965) 466-477.

NEIRA, E., "El problema de Dios en TC" REVISTA JAVERIANA 65 (1966) 235-247; idem en ESTUDIOS (Buenos Aires 1966) 276-285.

ÖBLİGÁDOO, A., "La antropología de TC" ESTUDIOS 54 (Buenos Aires 1965) $113-120$.

OLARTE, T., "El universö según TC" REV. FILOSOFICA DE COSTA RICA 1 (San José 1957) 137-148.

ÓLARTE, T., "El mensaje de TC" DIALOGOS 2 (Puerto Rico 1965) n. 4 $73-85$.

OLCOZ, I., "La actitud de y ante TC" SIC 32 (Caracas 1969) 211-213.

ORELLANA, M., "El método crítico de T" BOLETIN DE LA UNIV. DE CHILE 55 (Santigo de Chile 1965) 57-60.

ORESME, N., "Darwin y TC" ABSIDE 29 (México 1965) 336-341.

PARIS, C., "Unamuno y TC" CUADERNOS PARA EL DIALOGO 16 (Madrid 1965) 13-14.

PASCUAL MARINA, A., "TC y su visión del mundo" EIDOS 7 (Madrid 1961) $175-200$. 
PEREZ ARBELAEZ, E., "Desde el átomo al noon. Una crítica al PTC" REVISTA JAVERIANA 51 (Bogotá 1959) 58-67.

PEREZ DEL VISO, I., "Teilhard, sacerdote del Cristo cósmico" ESTUDIOS 54 (Buenos Aires 1965) 99-106.

" PHILIPPE DE LA TRINITE, "Teilhard de Chardin" EL CRUZADO ESPAÑOL 11 (Barcelona 1968) 61-66.

QUEROL, A. de, "Teilhard sigue señalando el punto Omega" EL CIERVO 182 (Barcelona 1969) 17.

RIAZA, F., "El hombre y el cosmos en la fenomenologia tellhardiana" HECHOS $Y$ DICHOS (Zaragoza 1966) 274-281.

RIAZA, F., "El hombre como fenómeno evolutivo según TC" ARBOR 67 Madrid 1967) 5-21.

RIAZA, F., "Aportaciones teilhardianas a una filosofia de la técnica" PENSAMIENTO 24 (Madrid 1968) 109-124.

RIAZA, F., "Teilhard y lo femenino" RAZON Y FE 178 (Madrid 1968) 309334.

* RIEDlinger, H., "El dominio cósmico de Cristo" CONCILIUM 2/1 (Madrid 1966) 108-126.

RIVERA DE VENTOSA, E., “¿Más luz en torno a TC?” AUGUSTINUS 11 (Madrid 1966) 417-422.

RODRIGUEZ, A., "La evolución, fenómeno cósmico en TC" "HORIZONTES 10 (Puerto Rico 1967) 45-59.

RODRIGUEZ ARAMBERRI, J., "Biografia completa de TC" LOS PROTAGONISTAS DE LA HISTORIA, n. ${ }^{\circ} 36$ (Madrid 1970) 28 pp.

ROIG GIRONELLA, J., "Sentido y alcance de la obra de Teilhard según sus criticos" ESPIRITU 13 (1964) 38-63; VERBO, III, 30, p. 523; CRISTIANDAD, 21, 177-180, 257-261; 22, 78-81.

ROLDAN, A., "La respuesta ignaciana a la crisis cientifico-filosôfica actual" RAZON Y FE 158 (Madrid 1958) 41-54 (sobre TC, 43-47).

RUBIO CARRACEDO, J., "Para leer a TC. La dialéctica teilhardiana" ESTUDIO AGUSTINIANO 2 (Valladolid 1967) 589-594.

R,UBIO CARRACEDO, J., "Quince años después. Del teilhardismo a Teilhar" ARBOR 76 (Madrid 1970), 43-46 (n. 297-8).

RUBIO CARRACEDO, J., "TC y su 'frente humano" ESTUDIO AGUSTINIANO 5 (Valladolid 1970), 373-392.

RUIZ ORTIZ, A., "Teilhard en 15 anécdotas" HECHOS Y DICHOS (Zaragoza 1966) 302-311.

* RUSSO, F., "El P. Teilhard de Chardin" AMERICA 103 (1960) 185-189; MENSAJE, XII (1960).

SALDARRIAGA, A., "La danza de las esferas o panorama del pensamiento de PTC" UNIV. DE ANTIOQUIA 43 (Medellin 1967) 245-319; 399$448 ; 44,3-73$.

SANJOSE, E., "Teilhard, acontecimiento espiritual del siglo" INCUNABLE 5 (Salamanca 1965) 507-512.

SANZ TENA, J. M., "Humanismo de TC", LICEO FRANCISCANO 19 (Santiago de Compostela 1966) 93-123. 
SCHMAUS, M., "En general hay que decir "si" a TC". INCUNABLE 192 (Salamanca 1965), 7.

SILES, J., "Las dos fuentes de la revolución contemporánea. El pensamiento filosófico y religioso de TC" FINIS TERRAE 9 (Santiago de Chile 1962) $86-94$.

SOBRINO, J., "TC en sus cartas" ESTUDIOS CENTROAMERICANOS 20 S. Salvador 1965) 223-228.

SOLA, F., "TC, ¿es Teilhard de Chardin?" ESPIRITU 14 (Barcelona 1965) $30-47$.

SOLAGUREN, C., "EI cristocentrismo cósmico de TC" VERDAD Y VIDA 19 (Madrid 1961) 131-143.

SOLAGUREN, C., "Ciencia, método y presupueștos en el pensamiento de TC" VERDAD Y VIDA, 20 (Madrid 1962) 509-531.

SOLAGUREN, C., "En torno a la 'pellgrosidad' de TC" VERDAD Y VIDA 21 Madrid 1963) 323-355.

SOLAGUREN, C., "La Cristología del PTC y el pricipio y fundamento de S. Ignacio" MANRESA 35 (Madrid 1963) 5-24.

SORIA, M., "Nuevo asedio a TC" PUNTA EUROPA 11 (Madrid 1966) n. 2 , 40-67.

SORIA, M., "Puntualizaciones sobre TC" PUNTA EUROPA 115 (Madrid 1967) $44-50$.

SORIA, M., “TC ¿científico o hereje?" NUEVA EPOCA (Madrid, 11-7-1964) 19-20.

SOUVIRON, J. M., "Una evolución en torno a la evolución" LA ESTAFETA LITERARIA 290 (Madrid 1964) 12-13.

* SPEAIGHT, R., "Teilhard y los jesuitas" RAZON Y FE 175 (Madrid 1967) $423-430$.

TOMAS CABOT, J., "El futuro del hombre. La teoría de TC" DESTINO 1502 (Barcelona 1966) 44-47.

TORRES ARIAS, R., "Hombre y mito" COMUNIDAD 2 (México 1967) 697701.

URDANOZ, T., "Dos obras de critica en torno a TC" ESTUDIOS FILOSOFICOS 15 (Santander, 1966) 137-147.

VALVERDE, C., "¿Qué hay que pensar sobre TC?” SAL TERRAE 51 (Santander 1963) 668-689.

VALVERDE, C., "Evolucionismo teilhardiano y 5.a vía". VI Congreso Tomista Internacional. Roma 1965. I, 295-301.

VALVERDE, C., "Recuerdo a Teilhard. A los diez años de su muerte" HUMANIDADES 17 (Comillas 1965) 380-386. Idem en REVISTA JAVERIANA 66 (Boootá 1966) 292-298.

VALVERDE, C., "La flecha de la evolución. Ooción humana y exigencia cósmica de lo divino en el pensamiento de TC" HUMANIDADES 18 (Comillas 1966) 377-386.

VALLS MASRIERA, C., "Sobre el pensamiento filosófico y teológico de PTC". ESPIRITU 12 (Barcelona 1963) 31-38. 
VELEZ CORREA, J., “Un jesuita evolucionista?” REVISTA JAVERIANA 51 (Bogotá 1959) 144-160.

VELEZ CORREA, J., "El pensamiento filosóflco en la teoria de TC" REVISTA JABERIANA 59 (Bogotá 1963) 156-175.

* WESPIN, D., de, "Cómo ser feliz según TC" INDICE 172 (Madrid 1963) 133.

WESPIN, D. de, "En el corazón del problema" INDICE 186 (Madrid 1964) 3-5.

ZAVALA, I. M., "La Materia en Marx y en TC" LA PALABRA Y EL HOMBRE 33 (Xalapa, Méx. 1965) 63-80.

ZOLLA, E., "A propósito de una más reciente publicación sobre TC" LA GACETA (Tucumán 30-3-1967).

ZOLLA ,E., "TC o del futurismo cósmico". LA GACETA (Tucumán, 12-31967).

4. Tesis todavia inéditas:

BRAJONES CUBILES, A., Lo sobrenatural en Teilhard. Univ. Pontif. de Salamanca. 1970 (todavía no defendida).

DIEZ RAVENA, A. V., Determinismo y libertad en la obra de PTC. Facultad de Fil. y Letras. Univ. de Madrid, 1965. $484 \mathrm{pp}$.

FIERRO, A., El proyecto teológico de TC. Facultad de teología de Zaragoza. 1966. $221 \mathrm{pp}$.

GARCIA ABRIL, G., Evolución y conciencia en PTC. Facultad de Fil. y Letras. Univ. de Barcelona, 1962. $89 \mathrm{pp}$.

LOPEZ ODIO, M. M., Concepción de la "materia" en la obra de PTC, "El Fenómeno Humano". 1961. 29 pp.

RUBIO CARRACEDO, J., La antropología educativa de TC. Facultad de Fil. y Letras. Univ. de Madrid. 1970. 545 pp.

SOTO, J. A., Función de la Reflexión en PTC. Univ. de Costa Rica 1961. $25 \mathrm{pp}$.

Ya en prensa el presente trabajo, ha aparecido el n. ${ }^{\circ}$ extraordinario que la revista "Pensamiento" (26, Madrid 1970, nn. 102-3) dedica a Teilhard como filósofo, con la aportación de algunos de los mejores especialistas españoles. Me limito a transcribir el indice: E. COLOMER, “TC, filósofo", 141-161; F. RIAZA, "Notas para un análisis formal de la fenomenologia teilhardiana", 163-179; C. PARIS, "Los aspectos cosmológicos en la obra de TC", 181-197; L. ENRIQUEZ, "Materiaespíritu en la visión antropológica teilhardiana", 199-211; J.S. LUCAS, "TC y el panteísmo", 213-230; C. VALVERDE, "TC y el marxismo", 231-253; L. MARTINEZ GOMEZ, "TC entre dos siglos", 255-275. 
Igualmente "Colligite" (16, León 1970, n. ${ }^{\circ}$ 64, 47-78) recoge algunos extractos sobre Teilhard de Mons. Garrone, S. de la Varga, P. Chauchard, E. del Río, F. Spadafora y E. Guerrero).

De otro lado, sería preferible no citar siquiera los vergonzosos alegatos de A. R. Villanova en los números 297-300 de "Cruzado Español".

José Rubio CarRacedo

Valladolid, 1970. 